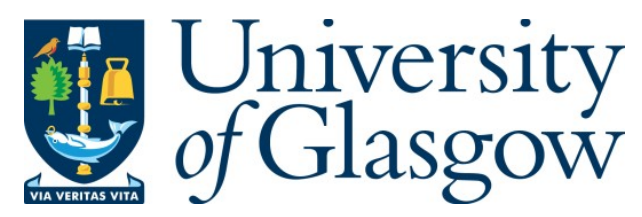

Levens, P.J., Schmieder, B., Labrosse, N., and Ariste, A. L. (2016) Structure of prominence legs: plasma and magnetic field. Astrophysical Journal, 818(1), 31.

There may be differences between this version and the published version. You are advised to consult the publisher's version if you wish to cite from it.

http://eprints.gla.ac.uk/121779/

Deposited on: 09 August 2016

Enlighten - Research publications by members of the University of Glasgow http://eprints.gla.ac.uk 
Draft Version December 16, 2015

Preprint typeset using LTEX style emulateapj v. 01/23/15

\title{
STRUCTURE OF PROMINENCE LEGS: PLASMA AND MAGNETIC FIELD
}

\author{
P. J. LeVENS \\ SUPA School of Physics and Astronomy, University of Glasgow, Glasgow, G12 8QQ, UK and \\ Observatoire de Paris, Meudon, 92195, France \\ B. SCHMIEDER \\ Observatoire de Paris, Meudon, 92195, France \\ N. LABRosSE \\ SUPA School of Physics and Astronomy, University of Glasgow, Glasgow, G12 8QQ, UK \\ AND \\ A. López Ariste \\ Institut de Recherche en Astrophysique et Planétologie, Toulouse, France \\ (Dated: Received ...; accepted ...) \\ Draft version December 16, 2015
}

\begin{abstract}
We investigate the properties of a 'solar tornado' observed on 15 July 2014, and aim to link the behaviour of the plasma to the internal magnetic field structure of the associated prominence. We made multi-wavelength observations with high spatial resolution and high cadence using SDO/AIA, the IRIS spectrograph and the Hinode/SOT instrument. Along with spectropolarimetry provided by the THEMIS telescope we have coverage of both optically thick emission lines and magnetic field information. AIA reveals that the two legs of the prominence are strongly absorbing structures which look like they are rotating, or oscillating in the plane of the sky. The two prominence legs, which are both very bright in Ca II (SOT), are not visible in the IRIS Mg II slit-jaw images. This is explained by the large optical thickness of the structures in $\mathrm{Mg}$ II which leads to reversed profiles, and hence to lower integrated intensities at these locations than in the surroundings. Using lines formed at temperatures lower than $1 \mathrm{MK}$, we measure relatively low Doppler shifts on the order of $\pm 10 \mathrm{~km} \mathrm{~s}^{-1}$ in the tornado-like structure. Between the two legs we see loops in Mg II, with material flowing from one leg to the other, as well as counterstreaming. It is difficult to interpret our data as showing two rotating, vertical structures which are unrelated to the loops. This kind of 'tornado' scenario does not fit with our observations. The magnetic field in the two legs of the prominence is found to be preferentially horizontal.
\end{abstract}

\section{INTRODUCTION}

The word 'tornado' has been used synonymously with prominences for many years (Pettit 1932, 1943), but the term has more recently been used again to describe specific phenomena in the solar atmosphere. Pike \& Mason (1998) used the term to describe macrospicules seen by the Solar and $\mathrm{He}$ liospheric Observatory (SOHO; Domingo et al. 1995), however not in relation to a prominence. It was only with the launch of the Solar Dynamics Observatory spacecraft and its high resolution imager, the Atmospheric Imaging Assembly (SDO, AIA; Lemen et al. 2012), that authors began writing of 'tornadoes' in relation to prominences again. Li et al. (2012) and Su et al. (2012) each used AIA images and techniques to demonstrate apparent rotation in prominence legs. Li et al. (2012) studied a tornado-like prominence with relation to the surrounding cavity, and found swirling motions and apparent flows in the prominence structure, and Panesar et al. (2013) investigated possible mechanisms for the observed motions and flows. Su et al. (2012) used time-distance diagrams, or 'stack-plots', using coronal filters from AIA to measure and quantify sinusoidal oscillations in tornadoes, finding that the measured period related to a rotational velocity of $6-8 \mathrm{~km} \mathrm{~s}^{-1}$.

The work of Wedemeyer-Böhm et al. (2012) was showing a swirling motion at different heights in the solar atmosphere, on disc, using both AIA and data from the Crisp Imaging Spectropolarimeter at the Swedish Solar Telescope (CRISP,

p.levens.1@ research.gla.ac.uk
SST; Scharmer et al. 2003). These 'chromospheric swirls' they found to originate in the chromosphere, and extend part of the way into the corona in the AIA channels. These 'magnetic tornadoes', however, do not appear to be related to any filamentary structure.

It is important here that we make a distinction between types of 'tornado' as referenced in the literature. These 'chromospheric swirls' have also been dubbed 'magnetic tornadoes' (Wedemeyer-Böhm et al.2012; Wedemeyer et al.2013), but do not necessarily relate to prominences/filaments. Those that are prominence-tornadoes have been called 'giant tornadoes' (Wedemeyer et al. 2013), as well as more generally 'solar tornadoes' (Li et al. |2012, Su et al. 2012; Panesar et al. 2013; Su et al. 2014; Levens et al. 2015), and it is these prominence-tornadoes that we are here referring to upon using this term. However, we must make a further distinction here. The 'solar tornadoes' which were studied in Su et al. (2012, 2014) and Levens et al. (2015) appear as absorbing features on the limb in AIA coronal images, whereas the one that was studied by Li et al. (2012) and Panesar et al. (2013) appears bright in AIA coronal channels. This distinction is made in the work of Panasenco et al. (2014), where they argue that these observational features can be explained by 2D oscillation or counterstreaming in prominence spines and barbs, and plasma motion along a writhed field respectively. It is the first type that we study here.

High resolution imaging can only build part of the picture of these structures. Spectroscopic techniques further our un- 
derstanding of the nature of these solar tornadoes, and have been used by a number of authors. Wedemeyer et al. (2013) presented the first Dopplergrams of such a structure using $\mathrm{H}$ $\alpha$ data from CRISP/SST, where they found a split Doppler pattern with blueshifts of $20 \mathrm{~km} \mathrm{~s}^{-1}$ to $30 \mathrm{~km} \mathrm{~s}^{-1}$ on one side of the base of the tornado, and redshifts of $10 \mathrm{~km} \mathrm{~s}^{-1}$ to $30 \mathrm{~km} \mathrm{~s}^{-1}$ on the opposite side. More recently the Extremeultraviolet Imaging Spectrometer (EIS; Culhane et al. 2007) on board the Hinode (Kosugi et al. 2007) satellite has been used to study tornadoes, where Su et al.(2014) found a similar split Doppler pattern to Wedemeyer et al. (2013), but this time in lines formed above $1.5 \mathrm{MK}$, with line-of-sight velocities of around $2-5 \mathrm{~km} \mathrm{~s}^{-1}$. This temperature range was extended down to $\sim 1 \mathrm{MK}$ by Levens et al. (2015). Su et al. found this antisymmetric Doppler pattern to be persistent for at least three hours of observation, which they and others (e.g. Orozco Suárez et al. 2012) have interpreted as rotation of the tornado structure about a central axis.

Other explanations for these observations have been put forward (see e.g. Panasenco et al.2014), with condensation along magnetic loops, or oscillation and counterstreaming in prominence barbs. Wedemeyer et al. identified giant tornadoes as the legs of prominences, which leads to the question: what is the magnetic structure of prominence legs? For active region filaments it appears that there is a common view that both ends of filaments are anchored in opposite sides of polarities along the polarity inversion line (PIL), with a spine making an angle of $25^{\circ}$ with the PIL. For quiescent filaments, lying between low magnetic field regions, the situation is not so clear, and is still debated (Mackay et al. 2010). The intermediate legs or feet would not be directly rooted in the polarities, but sustained in the dips of field lines of the magnetic rope of the filament, attracted towards the photosphere by parasitic polarities. In these regions the magnetic field is parallel to the photosphere, with the plasma in the dips (Aulanier \& Démoulin 1998; López Ariste et al. 2006; Dudík et al. 2008). Analysis of both the magnetic structure of tornadoes and the physical conditions of the plasma is necessary for understanding the apparent rotation mechanism of the tornado, and to discern whether or not it is really rotation that we are seeing.

During an international campaign in 2014, utilising both ground-based and space-based instruments, we focused on the observation of prominences. We have here selected one prominence, observed on July 15, 2014, for analysis. This prominence appears to have similar observational characteristics to those of a tornado.

In Section 2 we describe the instruments and observations: the Interface Region Imaging Spectrograph (IRIS; De Pontieu et al. 2014), the SOT instrument aboard Hinode, as well as the polarimeter at the Télescope Héliographique pour l'Etude du Magnétisme et des Instabilités Solaires (THEMIS, French telescope in the Canary Islands). Also in Section 2 we describe the prominence and tornado morphology as seen by SDO/AIA. Section 3 discusses the physical parameters obtained from the analysis of the data from these various telescopes and what they tell us about the nature of the prominence. Section 4 contains discussion of our results within the context of previous work. In Section 5 we present our conclusions.

\section{OBSERVATIONS}

The target prominence for July 15 was tracked as filament across the disc for a number of days by ground-based observa-

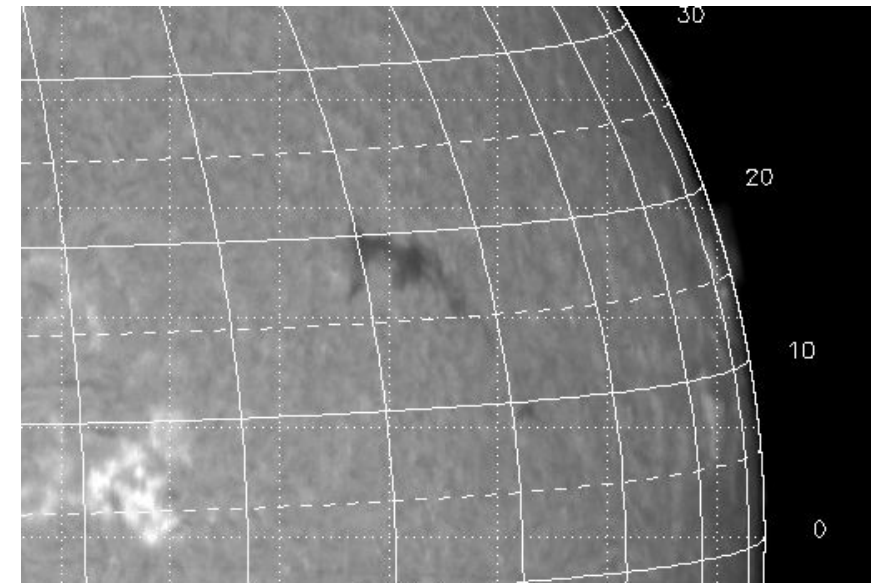

Figure 1. Filament observed at 13:37 UT on July 11 2014, in H- $\alpha$ with the Meudon survey instrument.
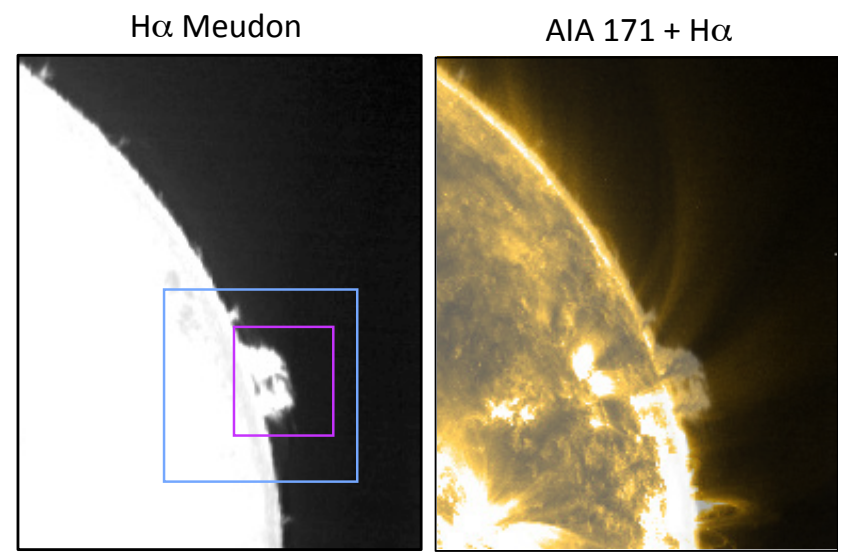

Figure 2. Left: H- $\alpha$ prominence observed at 09:40 UT on July 15, 2014 (Meudon survey). The purple and blue boxes show the fields of view in the AIA images of Figures 3 and 4 respectively. Right: AIA $171 \AA$ A overlaid with the $\mathrm{H}-\alpha$ prominence.

tories using H- $\alpha$ filters (e.g. from the Meudon survey, Figure 1. on July 11) before it became visible on the limb. Figure 2 (left panel) shows the prominence in $\mathrm{H}-\alpha$ on July 15, clearly showing two column-like structures which are bright in emission. The right panel of Figure 2 shows an overlay of the AIA $171 \AA$ image from the same time. Images made using coronal filters of AIA (Figure 3, left panel and online movie) show the prominence as two dark columns of material, absorbing the background emission, which display tornado-like behaviour when observed over time (see online movie accompanying Figure 3). These dark columns from AIA align with the bright columns in $\mathrm{H}-\alpha$ (Figure 2, right panel).

The prominence appears differently when viewed in optically thick lines formed at chromospheric and transition region temperatures. Figure 3, right panel, shows the $304 \AA$ filter from AIA, which is dominated by emission from the He II doublet at $303.78 \AA$. In this waveband we see much more clearly the extent of the prominence above the limb, as well as the horizontal structure in the prominence body, which is also seen in Ca II from Hinode/SOT (Figure 4 (b)) and $\mathrm{Mg}$ II from IRIS (Figure 4 (c)).

\subsection{Instruments}

\subsubsection{IRIS}



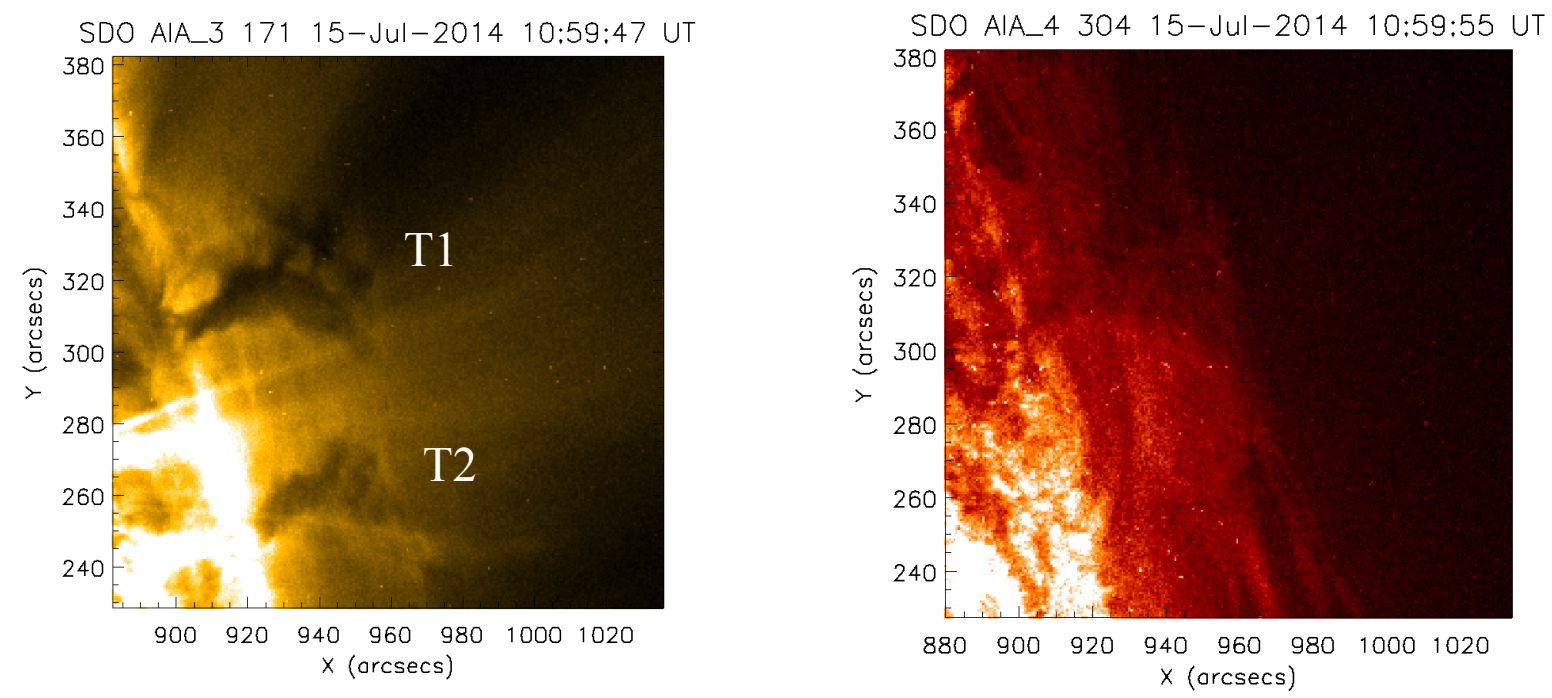

Figure 3. The prominence observed on July 15, 2014 by AIA in $171 \AA$ A (left) and $304 \AA$ (right). Overlayed on the left panel are the identifiers used to denote the two columns here - T1 and T2. Online movie shows the tornado-like nature of these two prominence legs in AIA $171 \AA$.

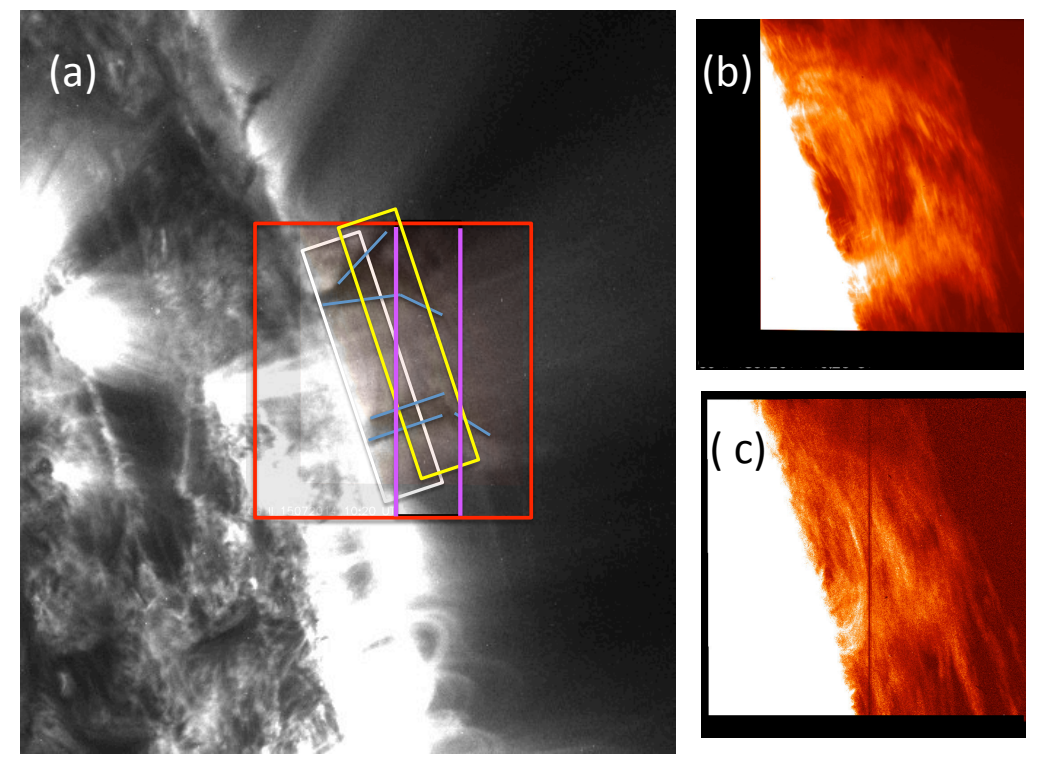

Figure 4. The coordinated prominence observation on 15 July 2014, here shown at 10:20 UT. (a) Two columns seen in absorption by AIA in the 171 A passband, with the field of view corresponding to that shown as a blue box in Figure 2 Overlayed are the field of view of IRIS and SOT (red box) and the fields of view of the two THEMIS rasters from this day (white and yellow boxes). The magenta box outlines the region covered by the IRIS raster. The blue lines indicate the edges of the two absorption features. Co-aligned images of the prominence are shown in (b), Ca II from Hinode/SOT, and (c), in Mg II from the IRIS slit-jaw using the $2796 \AA$ filter. The IRIS and SOT pointings were aligned by 2D cross correlation, using on-disc features. The images of SOT and IRIS (b and c respectively) are inserted into a black square, reflecting the red box of panel (a). 
IRIS performed a 16-step coarse raster observation from 08:00 to 11:00UT on July 15,2014 . The pointing of the telescope was $\left(940^{\prime \prime}, 284^{\prime \prime}\right)$, with spatial pixel size of $0.167^{\prime \prime}$. The raster cadence of the spectral observation in both the near ultraviolet (NUV, 2783 to $2834 \AA$ ) and far ultraviolet (FUV, 1332-1348 $\AA$ and 1390-1406 ̊) wavelength bands was 86 seconds. Exposure time was 5.4 seconds per slit position. Slit-jaw images (SJI) in the broadband filters $(2796 \AA$ and $1330 \AA$ ) were taken with a cadence of 11 seconds. The FOV was $30^{\prime \prime} \times 119^{\prime \prime}$ for the raster and $119^{\prime \prime} \times 119^{\prime \prime}$ for the SJI. Calibrated level 2 data is used for this analysis, with dark current subtraction, flat field correction, and geometrical correction having all been taken into account (De Pontieu et al. 2014).

We mainly used the $\mathrm{Mg}$ II k $2796.35 \AA$ and $\mathrm{Mg}$ II $\mathrm{h}$ $2803.5 \AA$ lines, along with the slit-jaw images in the $1330 \AA$ and $2796 \AA$ filters for this study. The $\mathrm{Mg}$ II $\mathrm{h}$ and $\mathrm{k}$ lines are formed at chromospheric plasma temperatures $\left(\sim 10^{4} \mathrm{~K}\right)$. The SJI $2796 \AA$ filter samples emission mainly from the $\mathrm{Mg} \mathrm{II} \mathrm{k}$ line, while emission in the $1330 \AA$ slit jaw is an integration of the FUV emission from within a range of about $40 \AA$, including the total emission of two C II lines and UV continuum. The co-alignment between the optical channels is achieved by comparing the positions of horizontal fiducial lines.

\subsubsection{Hinode/SOT}

The Hinode Solar Optical Telescope (SOT; Tsuneta et al. 2008, Suematsu et al. 2008) consists of a $50 \mathrm{~cm}$ diffractionlimited Gregorian telescope and a Focal Plane Package including the narrowband filtergraph (NFI), broadband filtergraph (BFI), the Stokes Spectro-Polarimeter, and Correlation Tracker (CT). For this study, images were taken with a $30 \mathrm{sec}-$ ond cadence in the $\mathrm{Ca}$ II $\mathrm{H}$ line at $3968.5 \AA$ using the BFI. The Ca II images have a pixel size of $0.109^{\prime \prime}$, with a field of view of $112^{\prime \prime} \times 112^{\prime \prime}$.

\subsubsection{THEMIS spectropolarimetry}

The slit of the THEMIS MulTi Raies (MTR) spectrograph (López Ariste et al. 2000) was orientated parallel to the limb for these observations. The observations consist of two successive rasters with 30 positions separated by $2^{\prime \prime}$. The first raster started at 14:41 UT, and the second began at 16:55 UT. The acquisition of the full raster takes more than one and a half hours. For an unknown reason the rasters each contain only 11 slit positions, covering a $22^{\prime \prime}$ region. The slit is $120^{\prime \prime}$ long, and the pixel size is $1^{\prime \prime}$. Due to the grid mode of the polarimeter, the images are obtained by taking exposures at two successive displacements of the grid along the slit in order to cover the full spectra of the 4 Stokes parameters in the $\mathrm{He} \mathrm{I}_{3}$ line (I, Q, U and V). The nature of this observing mode creates dark vertical bars in the images, which indicate the boundaries between grid placements.

The raw data of the THEMIS/MTR mode was reduced using the DeepStokes procedure (López Ariste et al. 2009). The details of this data reduction can be found in Schmieder et al. (2013, 2014b). The resulting Stokes profiles of each pixel were fed to an inversion code based on Principal Component Analysis (López Ariste \& Casini 2002; Casini et al. 2003). In this inversion technique the observed profiles are compared to those in a database containing 90000 profiles, which were generated with known models of the polarization profiles of the $\mathrm{He} \mathrm{I} \mathrm{D}$ line, taking into account the Hanle and Zeeman effects (López Ariste \& Casini 2002). The optical thickness of the $\mathrm{He} \mathrm{I} \mathrm{D}_{3}$ line in prominences is small (Labrosse \& Gouttebroze 2001, 2004), and this justifies the single scattering approximation used in the computation of the profiles. It is also worth remembering that polarization is only dependent on population imbalances inside the 5-level atomic model used for the calculations but not on the total atomic population of this model. This so-called non-LTE problem of the second kind (Landi Degl'Innocenti \& Landolfi 2004) is solved, but in the absence of important optical thickness there is no need to address other non-LTE effects.

Returning to the inversion process, the most similar profile in the database is kept as the solution, and the parameters of the model used in the computation are kept as the inferred vector magnetic field, height above the photosphere and scattering angle. As described by López Ariste \& Casini (2002), a set of the nearest models, including the nearest one given as solution, is used to determine, for each inverted point, error bars for each one of the parameters of the model. These error bars are explicitly computed as the standard deviation of each one of the parameters of the model in that set of models near to the solutions. Four factors contribute to these error bars: the noise in the observed profiles, the finite size of the database, the presence of inherent ambiguities (as e.g. the 180 degrees ambiguity in the case of the azimuth) and the ability of our model to reproduce the observations. Ambiguities are well-known and their impact has been studied by Casini et al. (2005). López Ariste \& Casini (2002) found the typical error bars coming from the two first issues, noise and finite size of the database. Of particular interest for the present work is the error bar they found for the inclination of the magnetic field, 10 degrees. Any inversion error in the retrieved inclination larger than 10 degrees can therefore only be attributed to the fourth cause: the insufficiency of the model to explain the observations. Schmieder et al. (2014a) and López Ariste (2014) already used this fact to look for complex magnetic topologies and we shall return to this point in Section 3.1 below.

\subsection{Morphology of the tornado 2.2.1. AIA, SOT and IRIS}

The SDO/AIA images for the event on July 152014 show different structures in different wavebands, seen in Figure 3 It is in the AIA coronal filters, such as the left panel of Figure 3 , that we see the apparent turning of these structures, leading to the impression of a "tornado" (see online movie associated with Figure 3 . In the $171 \AA$ images (Fig. 3. left panel) we see the tornadoes as 'silhouettes' against the hot ( $1 \mathrm{MK})$ background coronal emission. The orientation of these two columns on the limb is not as simple as it seems from this AIA image. The prominence is not orientated north-south on the limb, rather it appears to have more of an east-west orientation (as can be seen in Figure 1). The northern column (herein known as tornado 1, T1, as labelled in Figure 3, left panel) is located closer to the observer than the southern one (herein tornado 2, T2, as shown in the lower part of Figure 3. left panel). This can be seen in AIA movies of the days leading up to this observation, in which T2 clearly crosses the limb first. We also note that there are loops of hot material in front of tornado 2, slightly obscuring it in these images. This is especially clear in other AIA coronal images (e.g. 193 $\AA$ and $211 \AA$ ), where T2 is completely obscured by the hot foreground emission.

The northern of the two columns consists of a vertical pillar of $10^{\prime \prime}$ wide and $15^{\prime \prime}$ tall, extending towards $50^{\prime \prime}$ high with dif- 
ferent branches which are moving around and changing shape. These branches occupy an inverted cone which is $40^{\prime \prime}$ wide at the top. The southern column is a wide pillar, perpendicular to the limb (10" wide, $20^{\prime \prime}$ tall).

In the AIA $304 \AA$ image (Fig. 3 right panel) we see a different structure. The prominence is much more extended than those absorbing features seen in $171 \AA$ (Labrosse et al. 2011), and appears similar in $304 \AA$ emission to both IRIS and Hinode/SOT images. We also note that there is a darker structure in the $304 \AA$ image at the location of tornado 1 - additional absorption that is not visible in the IRIS Mg II images. We note that this extra absorption could be due to other, hotter lines in this AIA passband. Although this waveband is dominated by He II emission there are also a number of lines formed at coronal temperatures in the window, most notably $\mathrm{Si}$ XI at $303.3 \AA$, formed at $\log \mathrm{T}=6.2$ (Dere et al. 1997, Landi et al. 2012).

Figure 4(b) shows the prominence as seen by SOT in Ca II. In this image not only can we identify the two columns which are visible in AIA $171 \AA$, but we also see some loop-like structure, similar to that seen in AIA $304 \AA$ (Fig. 3 , right panel) and the IRIS Mg II SJI (Fig. 4(c)).

In Figure 4(c) we show the IRIS slit-jaw image of the prominence using the $2796 \AA$ filter. The red box in Figure 4 (a) indicates the placement of the IRIS SJI with respect to the background AIA $171 \AA$ image, and the magenta box shows the rastering region that was covered during the study. The two tornadoes are not clearly visible in this waveband. This will be discussed in Section 3.2.1 after inspecting the spectra - both $\mathrm{Mg}$ II and Ca II lines have a large optical thickness compared to $\mathrm{H}-\alpha$.

\subsubsection{Time-distance analysis}

As was mentioned in Section 1, Su et al. (2012) used timedistance plots to measure sinusoidal oscillations in tornadolike prominences. In that paper they found a period that would relate to a rotational velocity of $6-8 \mathrm{~km} \mathrm{~s}^{-1}$. We apply a similar method here to the two tornadoes in the AIA $171 \AA$ image, the results of which are presented in Figure 5. Here we have taken two cuts through each of the columns, parallel to the limb, one at the base, and one near the top of the absorbing feature.

We selected different cuts across the two columns, parallel to the limb, to perform the time-distance analysis. The lower set of cuts are approximately $10^{\prime \prime}$ above the limb, with the upper cuts at $35^{\prime \prime}$ from the limb. The cuts through the lower part of each of the columns do not appear to display any oscillatory motion in the plane of the sky, or at least there is not enough contrast in the AIA images to measure. The upper parts of both columns, however, display a much clearer sinusoidal pattern. Taking a rough estimate at the period $(\sim 1.25$ hours) and amplitude $\left(\sim 10^{\prime \prime}\right)$ of these oscillations, we recover a velocity on the order of $10 \mathrm{~km} \mathrm{~s}^{-1}$, which is consistent with the results of Su et al. (2012), if we assume that the tornado is rotating

However, as has been pointed out by Panasenco et al. (2014), time-distance analysis like this does not prove that the observed structure is rotating. These observational signatures could also be caused by oscillations of plasma in the magnetic field, projected onto the plane of the sky. We cannot rule out this explanation with this data set, as will be discussed in the following analysis of THEMIS and IRIS data.
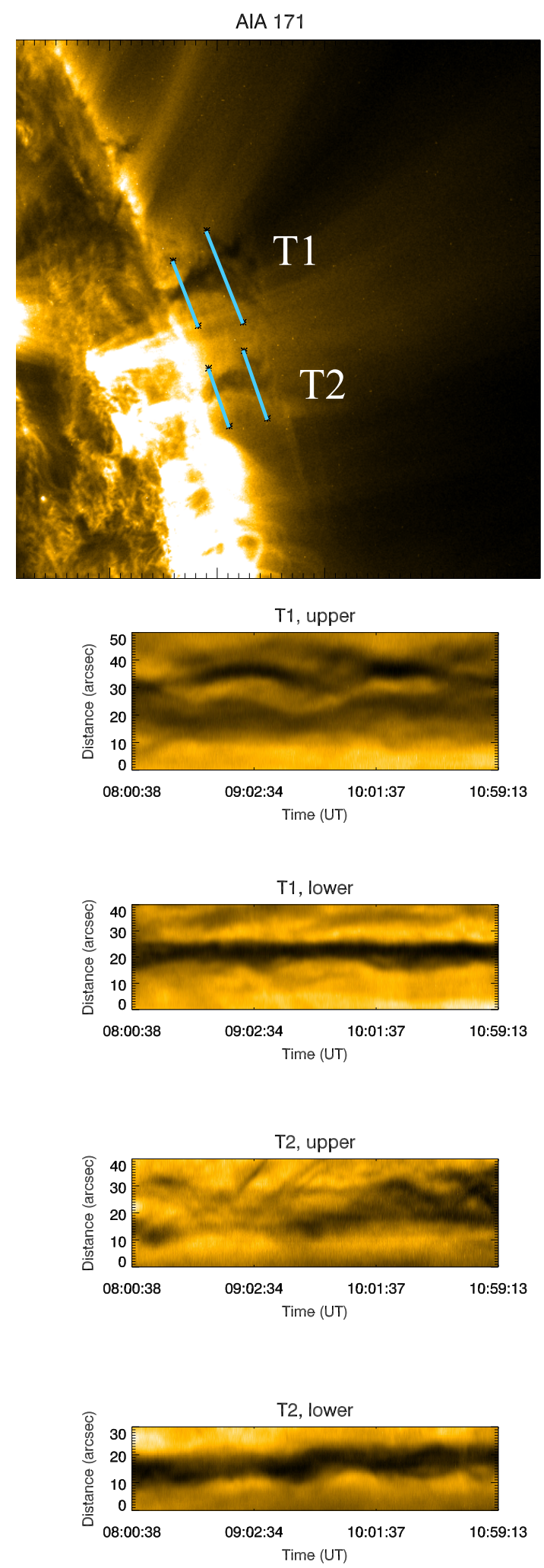

Figure 5. Time-slice diagrams for the two tornadoes, $\mathrm{T} 1$ in the north and T2 in the south, using the AIA $171 \AA$ waveband. The top image was taken at 11:00 UT, corresponding to the end time of the IRIS observation. Here we have taken two parallel cuts through each column: a lower cut near the limb and an upper cut which is nearer the top of each tornado, and these are shown in blue in the top panel. 


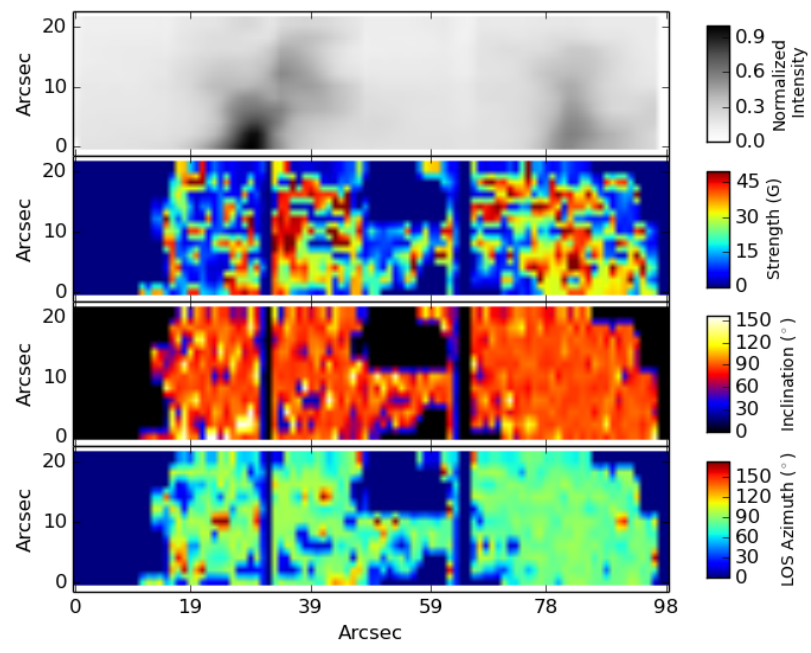

Figure 6. THEMIS observation of the prominence on July 15, 2014 between 16:55 UT and 18:10 UT in $\mathrm{He}_{\mathrm{I}} \mathrm{D}_{3}$ : (from top to bottom) intensity, magnetic field strength, inclination and azimuth.

\section{PHYSICAL PARAMETERS}

\subsection{Magnetic field vector}

The $\mathrm{He} \mathrm{I} \mathrm{D}_{3} 5876 \AA$ line appears as a doublet. The two spectroscopic components have a different sensitivity to the Hanle effect and are far from being saturated. This helps with constraining the two ambiguities that need to be resolved: the 180 degree ambiguity of the Zeeman effect and the 90 degree ambiguity of the Hanle effect. The first raster overlays the bottom $22^{\prime \prime}$ of the two columns, while the second raster is aimed at the top, with a slight overlap with the first raster - see Figure 4 (a), where the upper and lower THEMIS raster positions are represented by white and yellow boxes respectively.

Figure 6 presents the maps obtained after the inversion of the Stokes parameters recorded in the $\mathrm{He} \mathrm{I}_{3}$ line: (from top to bottom) Intensity, magnetic field strength, inclination, and azimuth for the scan represented by the yellow box in Figure 4 (observed between 16:55 UT and 18:10 UT). The origin angle of inclination is the local vertical, and the origin of the azimuth is the line of sight (LOS) in a plane containing the LOS and the local vertical. We see that the brightest parts of the prominence have a mean inclination of $90^{\circ}$ which means that the magnetic field in these parts is horizontal. However there is a large dispersion of the values $\left( \pm 30^{\circ}\right)$ from one pixel to the next in the lateral part of the prominence. Also in these bright parts of the prominence we find the strongest magnetic fields, ranging from around $20 \mathrm{G}$ up to $50 \mathrm{G}$ in some regions. We recover l.o.s. azimuth values of between $70^{\circ}$ and $100^{\circ}$. We note there is some structure between the columns, visible in the bottom three panels of Figure 6 seemingly joining the columns. In the intensity map, this structure is very dim, and is not visible without over-saturating the main prominence pillars. It is not certain that this faint structure is part of the prominence, or if it is plasma sitting in the foreground or background of the prominence that we are studying. It does not, however, appear to play any role in the inversions that we are interested in here, which is those from the columns themselves.

We have computed the histogram of the inclination for all points in the tornado, shown in Figure 7. The main peak of the distribution is centered on $90^{\circ}$, indicating a horizontal field

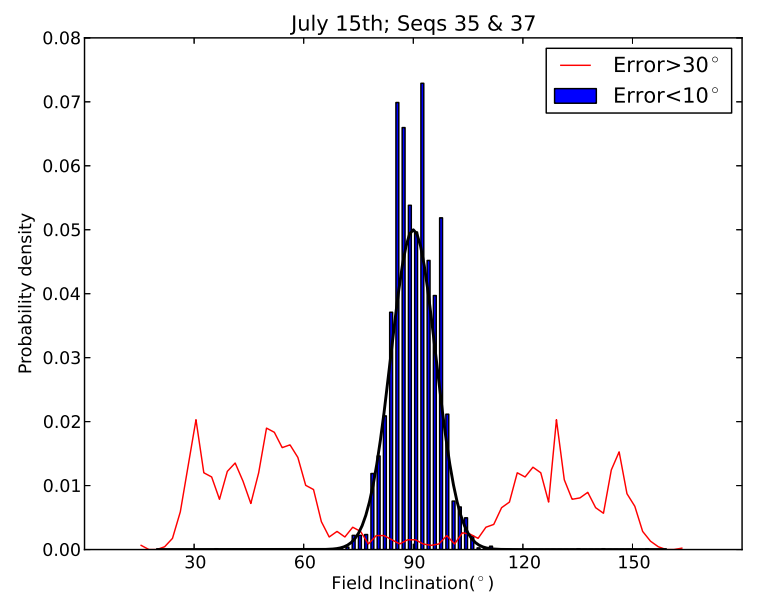

Figure 7. Histogram of magnetic field inclination for points in the prominence. The blue histogram indicates points whose error is $<10^{\circ}$, with the black line is a Gaussian with FWHM $=10^{\circ}$. The red line indicates the inclination of points where the error is $>30^{\circ}$.

with respect to the local limb. Those results have small error bars of 10 degrees (the black line is a Gaussian curve with standard deviation of 10 degrees that correctly reproduces the data distribution). As described above, this error bar is consistent with the expected errors from noise and the finite size of the database. We are confident that the magnetic field of the prominence is correctly measured in those cases. There are also two secondary peaks in Figure 7, which are wide from $30^{\circ}$ to $60^{\circ}$ and from $120^{\circ}$ to $150^{\circ}$ - with large error bars $\left(>30^{\circ}\right)$. These large error bars cannot be attributed either to noise, the finite size of the database or ambiguities. The inclinations of $60^{\circ}$ and $120^{\circ}$ have already been detected in prominences (Schmieder et al. 2014a; López Ariste 2014). These inclinations have been explained by the superposition of two magnetic fields: one horizontal and the other turbulent. This magnetic topology is not considered in the database used for inversion that includes just one magnetic field vector which is constant over the pixel. The inversion code tries to fit with a spread of models resulting in such error bars in these cases. The inclinations of $30^{\circ}$ and $150^{\circ}$, on the other hand, have also been observed in prominences (Schmieder et al. 2014b). We concluded from this that the magnetic field model of one field per pixel used by the PCA inversion code is not valid. We can definitely exclude a vertical field as a solution, as this would have been correctly inverted. Several possibilities that mix a number of magnetic components are yet to be explored.

\subsection{Spectral analysis of IRIS}

The main spectral lines that are visible in the IRIS spectra are the $\mathrm{Mg}$ II k and h lines at 2796.35 $\AA$ and 2803.50 A respectively. Other lines, such as the Si IV $1393.78 \AA$ line and the C II $1335.71 \AA$ line, appear very faint, with a low signal-tonoise ratio caused by the relatively short exposure time used in the study. We can, however, regain some information from these lines by averaging the spectral profiles along each slit over time. Figure 8 shows intensity as a function of slit position for the Mg II k, Si IV 1393.71 $\AA$ and C II 1335.71 $\AA$ lines after the profiles have been averaged for an hour of data.

Performing this average we see that the $\mathrm{Mg}$ II $\mathrm{k}$ line presents a very smooth profile up the slit, caused by the already strong SNR of this line. The other two lines present 

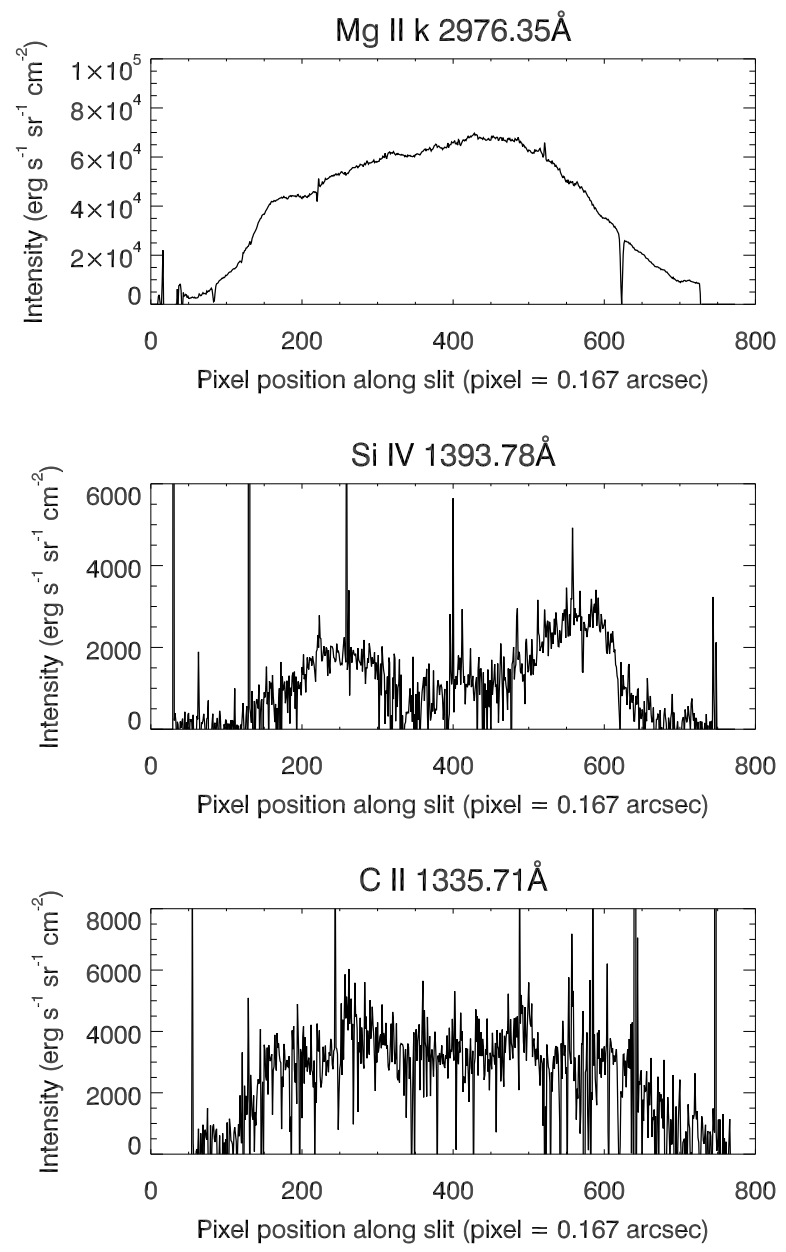

Figure 8. Intensity as a function of slit position for three IRIS spectral lines from the raster - Mg II k $2796.35 \AA$ (top), Si IV $1393.78 \AA$ A (middle) and C II $1335.71 \AA$ (bottom). These have all been averaged over an hour's worth of data in order to combat the poor signal-to-noise ratio of the Si IV and C II lines. Also note the differences in scale on the y-axes. These are all taken from the third IRIS slit position. In these plots pixel position 0 is to the south. The tornadoes T1 and T2 are located between pixels 535 and 650, and 190 and 260 respectively. Sudden drops in intensity approximately at pixels 90 and 620, seen most clearly in Mg II, are due to the fiducial lines on the IRIS CCDs.

noisier profiles, but with an hours worth of data we begin to see patterns emerging. Si IV presents two bright columns, cospatial with the dark silhouettes seen in AIA (Figure 3, left panel) and the brightenings seen in Hinode/SOT Ca II images (Figures 4 and 9 ). In the $\mathrm{C}$ II line we see a similar profile to that of $\mathrm{Mg}$ II.

We have analysed the Mg II spectra for the raster beginning at 10:21 UT, at each of the 16 slit positions. This time was selected as it coincides with the start of the Hinode observing time, giving us simultaneous IRIS and SOT observations. We can identify the two columns that correspond to the brightest regions in IRIS Si IV $1393.78 \AA$ (Figure 8 ) and SOT Ca II $3968.5 \AA$ emission (Figure 9), allowing us to locate them in IRIS Mg II images and spectra.

Figure 10 shows example spectra of the $\mathrm{Mg}$ II $\mathrm{k}$ line for ten slit positions taken from the raster at 10:21 UT. We note that the profiles are mostly reversed or have a flat top, especially in the locations identified as being co-spatial with the tornadoes

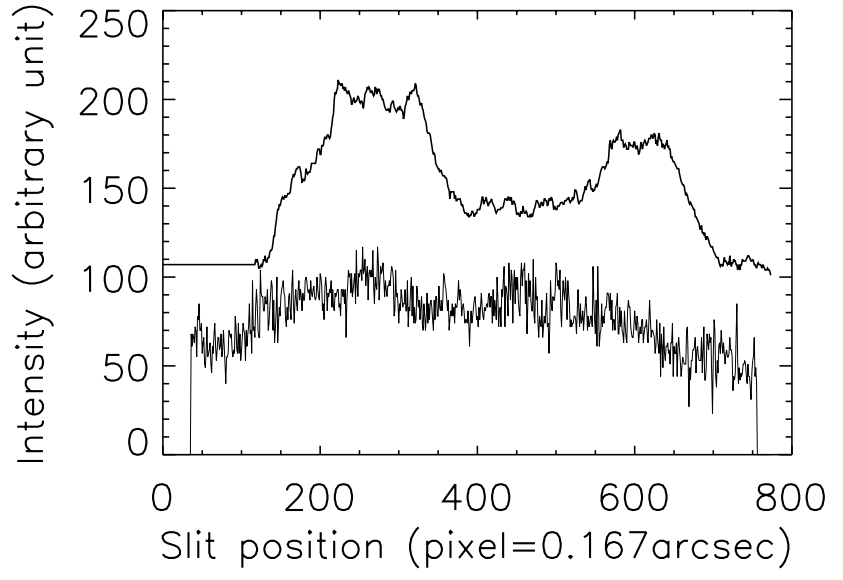

Figure 9. Intensity as a function of slit position for IRIS Mg II $\mathrm{k}$ (lower curve) and SOT Ca II H. These are both vertical cuts through the images taken at the time of the first IRIS slit position, with the IRIS profile here coming from the slit-jaw image. The tornadoes $\mathrm{T} 1$ and $\mathrm{T} 2$ are located between pixels 535 and 650, and 190 and 260 respectively.

(see Figure 11 and online movie).

Assuming a single Gaussian fit, we have computed the characteristics of the profiles for the two lines (Figure 12). The full widths at half maximum (FWHMs) are identical and the Doppler shifts are very similar, however the Doppler shifts between $\pm 5 \mathrm{~km} \mathrm{~s}^{-1}$ are slightly higher for the $\mathrm{h}$ line. The FWHM values fall in a range between $0.3 \AA$ and $0.4 \AA$ with some minima $(\sim 0.18 \AA)$ in the top and the bottom of the slit where the profiles are not reversed. The integrated intensities are around $4 \times 10^{4} \mathrm{erg} \mathrm{s}^{-1} \mathrm{sr}^{-1} \mathrm{~cm}^{-2}$ for the $\mathrm{k}$ line and around $3 \times 10^{4} \mathrm{erg} \mathrm{s}^{-1} \mathrm{sr}^{-1} \mathrm{~cm}^{-2}$ for the $\mathrm{h}$ line. In T1, which corresponds to the top of the slit, the intensities decrease over a short distance to $1 \times 10^{4} \mathrm{erg} \mathrm{s}^{-1} \mathrm{sr}^{-1} \mathrm{~cm}^{-2}$. The brightest parts of the spectra correspond to the loops between the two columns. Slit 1 crosses the south column (T2) close to the limb between pixels 180 and 230, the north column near the top (T1) between pixel 570 and 630 .

Figure 11 shows examples of the $\mathrm{Mg}$ II profiles in $\mathrm{T} 1$ and T2 corresponding to slit positions 0,8 and 15 . The T1 profiles are reversed, whereas the T2 profiles show a mixture of reversed and flat-topped profiles. Table 1 shows a number of derived parameters from the line profiles of the $\mathrm{Mg}$ II lines, derived using the analysis of the moments of the Mg II distribution. The maximum of the ratio $I_{\text {peak }} / I_{0}$ is found to be around 2.6 for the $\mathrm{k}$ line, with a corresponding integrated intensity of $4.5 \times 10^{4} \mathrm{erg} \mathrm{s}^{-1} \mathrm{sr}^{-1} \mathrm{~cm}^{-2}$. The T2 profiles are less reversed $(\max =1.4)$ and have higher integrated intensities of around $4.3 \times 10^{4} \mathrm{erg} \mathrm{s}^{-1} \mathrm{sr}^{-1} \mathrm{~cm}^{-2}$.

\subsubsection{Gas pressure and density}

With this information we are able to discuss the gas pressure using the table of Heinzel et al. (2014) for isobaric 1D models. For T1 some models could be considered with a pressure reaching 0.5 dyne $\mathrm{cm}^{-2}$, temperatures of $6000 \mathrm{~K}$ and with a slab thickness of $1000 \mathrm{~km}$. This would lead to an optical thickness of 820 . With a lower pressure $p=0.1$ dyne $\mathrm{cm}^{-2}$ and a temperature of $8000 \mathrm{~K}$ the optical thickness could be half (460) and the geometrical depth $5000 \mathrm{~km}$. This is a reasonable scenario, because we see strong absorption in the AIA $171 \AA ̊$ Amages.

High optical thickness is consistent with reversal of profiles. 


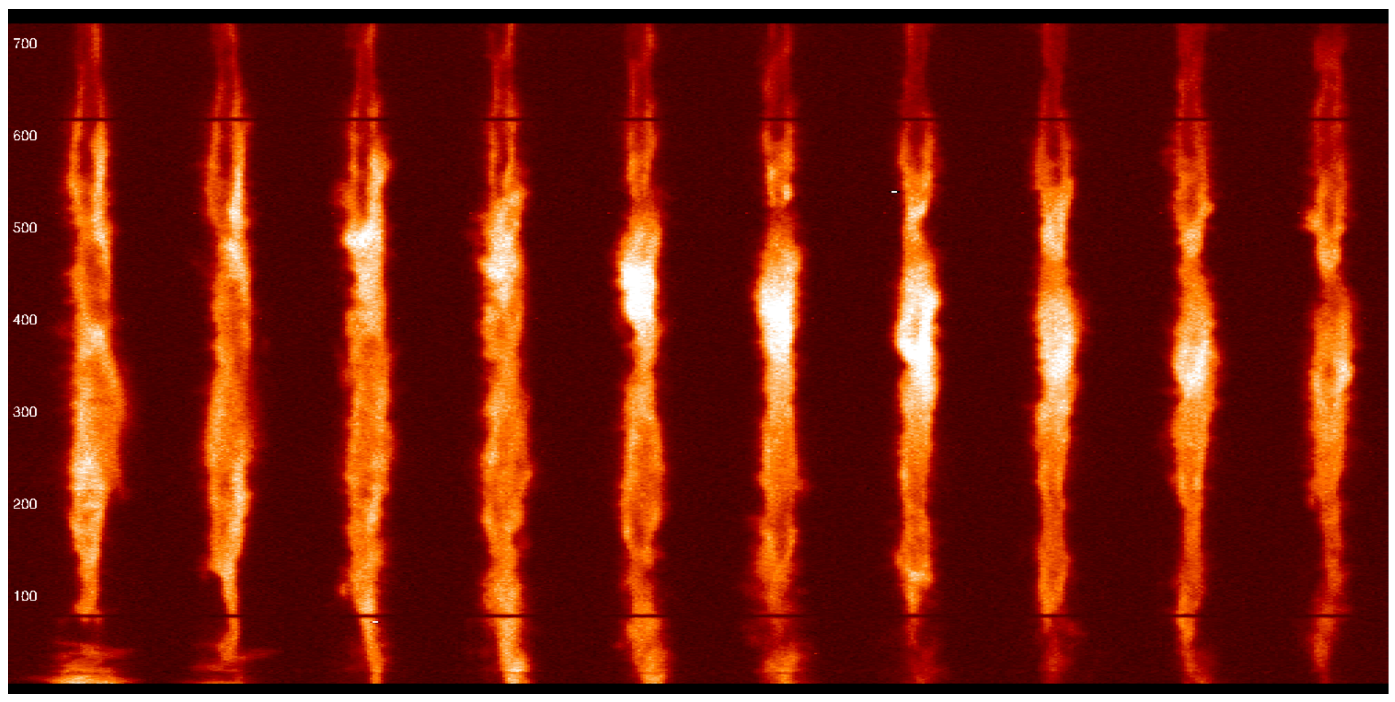

Figure 10. Example Mg II k spectra from the raster starting at 10:21 UT. Shown here are the first 10 slit positions of the raster, with the left-most slit position being closest to the limb. The scale on the left indicates pixel number, each pixel has a size of $0.167 \AA$. The horizontal dark lines through the slits approximately at pixels 90 and 620 seen here are due to the fiducial lines on the IRIS CCD. The tornadoes T1 and T2 are located between pixels 535 and 650 , and 190 and 260 respectively.
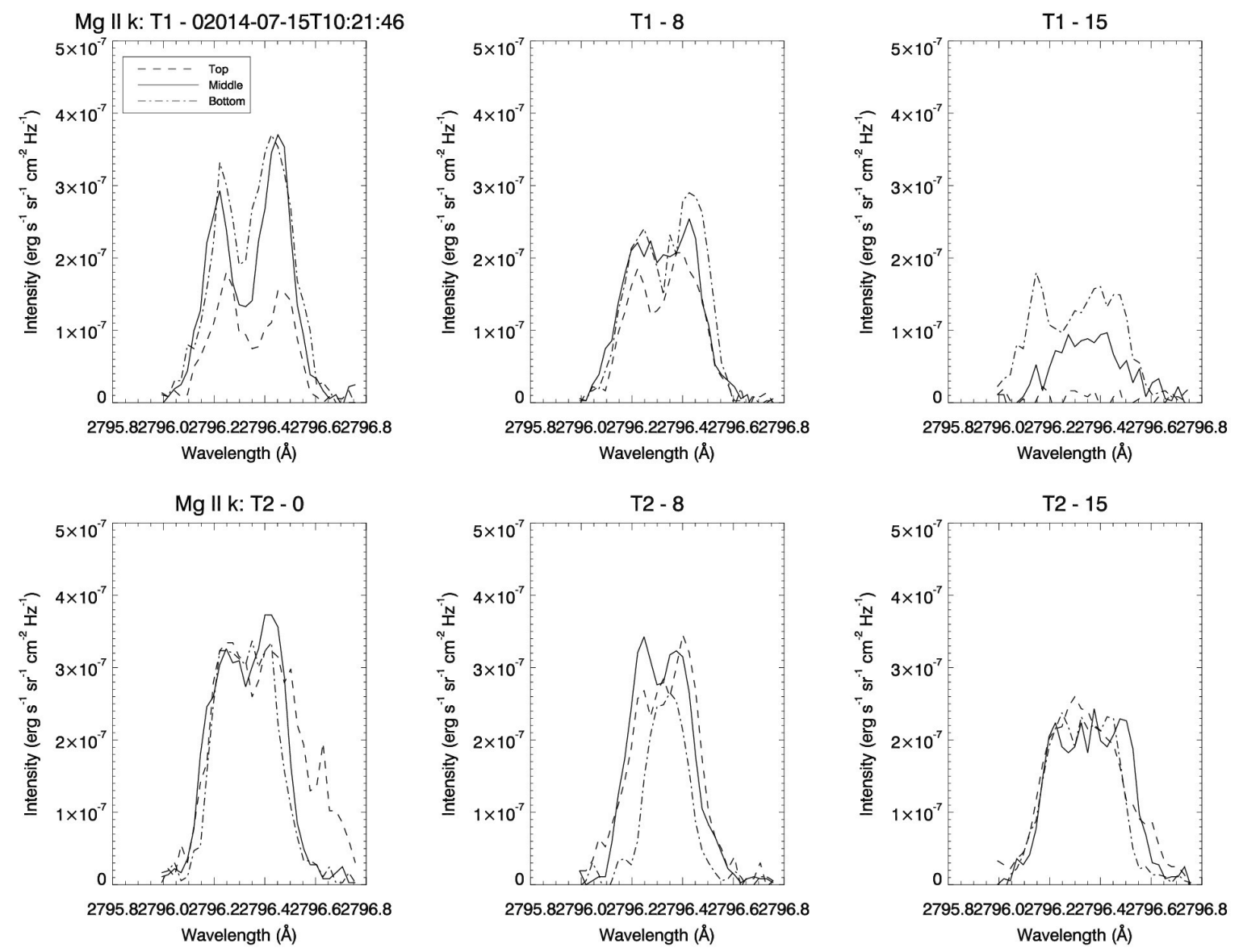

Figure 11. Line profiles of the IRIS Mg II k line at 10:21 UT: Top row - spectra at three pixel locations corresponding to tornado 1, in slits 0 (left), 8 (centre) and 15 (right). Bottom row - similar for tornado 2. Online movie shows the evolution of these profiles over time for the three hours of observation. 

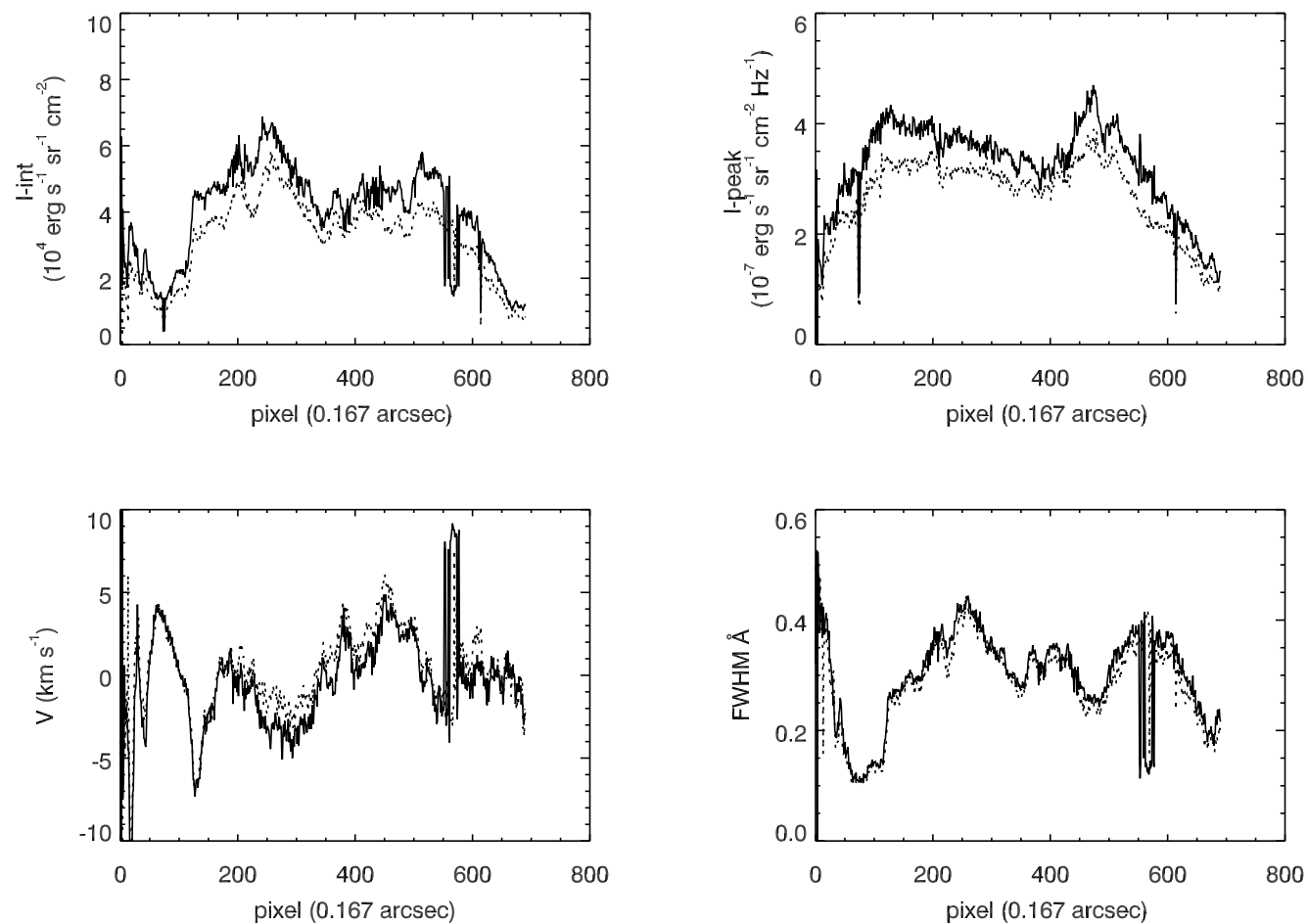

Figure 12. Fit parameters of the $\mathrm{Mg} \mathrm{II} \mathrm{h}$ and $\mathrm{k}$ lines along slit position 1, assuming Gaussian profiles: integrated intensity, peak intensity, line-of-sight velocity and FWHM. Solid lines are for Mg II k, dashed lines for Mg II h. The tornadoes T1 and T2 are located between pixels 535 and 650 , and 190 and 260 respectively.

Table 1

Line parameters measured for the IRIS Mg II k and h lines. Peak intensity has units $\mathrm{erg} \mathrm{s}^{-1} \mathrm{sr}^{-1} \mathrm{~cm}^{-2} \mathrm{~Hz}^{-1}$, and integrated intensity has units erg s $\mathrm{s}^{-1} \mathrm{sr}^{-1} \mathrm{~cm}^{-2}$. FWHM is in $\AA$. For reversed profiles, $I_{\text {peak }} / I_{0}$ is defined as the ratio of the peak intensity to the intensity at the reversal minimum. Also shown here are values of line-of-sight velocity, $\mathrm{v}_{\text {los }}$ with units in $\mathrm{km} \mathrm{s}^{-1}$, as derived from a single Gaussian fit to the $\mathrm{Mg}$ II $\mathrm{k}$ line.

\begin{tabular}{|c|c|c|c|c|c|c|c|c|c|c|}
\hline \multirow[t]{2}{*}{$\mathrm{Mg}$ II k } & \multirow[t]{2}{*}{ Slit } & \multicolumn{3}{|c|}{0} & \multicolumn{3}{|c|}{8} & \multicolumn{3}{|c|}{15} \\
\hline & & North & Centre & South & North & Centre & South & North & Centre & South \\
\hline \multirow[t]{6}{*}{ T1 } & $\mathrm{I}_{\text {peak }}\left(\times 10^{-7}\right)$ & 2.00 & 4.15 & 4.15 & 2.32 & 2.84 & 3.25 & 0.58 & 1.08 & 2.01 \\
\hline & $\mathrm{I}_{\text {peak }} / \mathrm{I}_{0}$ & 2.41 & 2.63 & 1.89 & 1.70 & 1.95 & 1.25 & 1.72 & 1.17 & 1.62 \\
\hline & $\mathrm{I}_{\text {int }}\left(\times 10^{4}\right)$ & 2.22 & 4.48 & 5.13 & 2.80 & 3.62 & 4.01 & 0.43 & 1.36 & 2.81 \\
\hline & FWHM & 0.31 & 0.33 & 0.31 & 0.30 & 0.29 & 0.30 & 0.08 & 0.31 & 0.35 \\
\hline & $\mathrm{I}_{\mathrm{k}} / \mathrm{I}_{\mathrm{h}}$ & 1.39 & 1.37 & 1.41 & 1.37 & 1.39 & 1.35 & 1.16 & 1.75 & 1.37 \\
\hline & $\mathrm{v}_{\mathrm{los}}$ & -1.19 & 0.88 & 1.57 & -1.70 & -3.53 & -0.09 & -7.65 & 0.17 & -4.58 \\
\hline \multirow[t]{6}{*}{$\mathrm{T} 2$} & $\mathrm{I}_{\text {peak }}\left(\times 10^{-7}\right)$ & 3.74 & 4.18 & 3.77 & 3.87 & 3.83 & 2.97 & 2.91 & 2.72 & 2.66 \\
\hline & $\mathrm{I}_{\text {peak }} / \mathrm{I}_{0}$ & 1.29 & 1.36 & $1.01(\mathrm{nr})$ & 1.21 & 1.23 & $1.00(\mathrm{nr})$ & $1.00(\mathrm{nr})$ & 1.33 & 1.25 \\
\hline & $\mathrm{I}_{\text {int }}\left(\times 10^{4}\right)$ & 6.33 & 5.28 & 4.56 & 4.39 & 4.32 & 2.40 & 4.06 & 3.86 & 3.54 \\
\hline & FWHM & 0.39 & 0.30 & 0.28 & 0.30 & 0.27 & 0.25 & 0.33 & 0.33 & 0.30 \\
\hline & $\mathrm{I}_{\mathrm{k}} / \mathrm{I}_{\mathrm{h}}$ & 1.39 & 1.41 & 1.39 & 1.45 & 1.37 & 1.49 & 1.33 & 1.25 & 1.30 \\
\hline & $\mathrm{v}_{\text {los }}$ & 2.28 & -1.61 & -2.68 & -1.57 & -4.04 & -1.23 & -2.10 & 0.87 & -2.46 \\
\hline \multirow[t]{2}{*}{$\overline{\mathrm{Mg} \text { II h }}$} & Slit & & 0 & & & 8 & & & 15 & \\
\hline & & North & Centre & South & North & Centre & South & North & Centre & South \\
\hline \multirow[t]{5}{*}{ T1 } & $\mathrm{I}_{\text {peak }}\left(\times 10^{-7}\right)$ & 1.46 & 3.63 & 2.98 & 1.78 & 2.28 & 2.70 & 0.50 & 0.70 & 1.40 \\
\hline & $\mathrm{I}_{\text {peak }} / \mathrm{I}_{0}$ & 2.42 & 3.21 & 1.68 & 1.40 & 1.26 & 1.64 & $\mathrm{n} / \mathrm{a}$ & 1.29 & 1.63 \\
\hline & $\mathrm{I}_{\text {int }}\left(\times 10^{4}\right)$ & 1.60 & 3.27 & 3.63 & 2.04 & 2.61 & 2.97 & 0.37 & 0.77 & 2.06 \\
\hline & FWHM & 0.35 & 0.30 & 0.30 & 0.26 & 0.28 & 0.25 & 0.41 & 0.35 & 0.33 \\
\hline & $\mathrm{I}_{\mathrm{k}} / \mathrm{I}_{\mathrm{h}}$ & 1.39 & 1.37 & 1.41 & 1.37 & 1.39 & 1.35 & 1.16 & 1.75 & 1.37 \\
\hline \multirow[t]{5}{*}{$\mathrm{T} 2$} & $\mathrm{I}_{\text {peak }}\left(\times 10^{-7}\right)$ & 2.92 & 3.11 & 3.20 & 2.89 & 2.89 & 2.44 & 2.41 & 2.22 & 2.63 \\
\hline & $\mathrm{I}_{\text {peak }} / \mathrm{I}_{0}$ & 1.07 & 1.21 & 1.12 & 1.33 & 1.42 & $1.00(\mathrm{nr})$ & 1.19 & 1.32 & 1.57 \\
\hline & $\mathrm{I}_{\text {int }}\left(\times 10^{4}\right)$ & 4.57 & 3.76 & 3.30 & 3.05 & 3.17 & 1.61 & 3.05 & 3.09 & 2.74 \\
\hline & FWHM & 0.34 & 0.24 & 0.23 & 0.24 & 0.28 & 0.24 & 0.30 & 0.29 & 0.27 \\
\hline & $\mathrm{I}_{\mathrm{k}} / \mathrm{I}_{\mathrm{h}}$ & 1.39 & 1.41 & 1.39 & 1.45 & 1.37 & 1.49 & 1.33 & 1.25 & 1.30 \\
\hline
\end{tabular}


The large absorption, or reversal, in the centre of the $\mathrm{Mg}$ II $\mathrm{h}$ and $\mathrm{k}$ lines reduces the integrated intensity values relative to what they would be if the profiles were not reversed. This explains why $\mathrm{T} 1$, when viewed in the IRIS slit jaw, is not as bright as it appears in the SOT Ca II images. For $\mathrm{H}-\alpha, \mathrm{Ca}$ and $\mathrm{Mg}$ with respective optical thicknesses $\tau_{\mathrm{H}-\alpha}, \tau_{\mathrm{Ca}}$ and $\tau_{\mathrm{Mg}}$, when we have $\tau_{\mathrm{H}-\alpha}=1$ we expect $\tau_{\mathrm{Ca}}$ to be 40 and $\tau_{\mathrm{Mg}}$ to be 70 (P. Heinzel, private communication). For He II $304 \AA$ we expect $\tau_{304} \simeq 280$ (with $\mathrm{T}=6000-8000 \mathrm{~K}, \mathrm{p}=0.1$ dyne $\mathrm{cm}^{-2}$, slab width $=1000 \mathrm{~km}$ ). This means that the line-ofsight emission in $\mathrm{H}-\alpha$ is the sum of the emission from all the different structures along the line of sight, while in the other lines what we see is mainly what is in the frontmost part.

In T2 there is generally less reversal in the profiles, with some even appearing non-reversed. Comparing these to the table of Heinzel et al. (2014) leads us to a low gas pressure scenario, with values of around 0.1 dyne $\mathrm{cm}^{-2}$. However this is not an acceptable solution because, as mentioned previously, both the absorption in $171 \AA$ and the emission in $\mathrm{Ca} \mathrm{II}$ and $\mathrm{He} \mathrm{I} \mathrm{D}_{3}$ lines are strong. Instead of the $1 \mathrm{D}$, isobaric case of Heinzel et al. (2014) we have to consider 2D models with a prominence-to-corona transition region (PCTR), as was outlined in Heinzel et al. (2015). In that paper, the authors showed that these models allow us to fit $\mathrm{H}-\alpha$ and $\mathrm{Mg}$ II lines with a relatively high pressure. Some of the profiles could also be interpreted by fitting with multiple Gaussians. This would indicate that the line of sight is crossing several structures, each with a different velocity - as was shown in Schmieder et al. (2014b).

\subsubsection{Doppler shifts}

Assuming Gaussian profiles for the IRIS Mg II lines, we return relatively small line-of-sight velocities $\left( \pm 5 \mathrm{~km} \mathrm{~s}^{-1}\right)$. The velocity pattern obtained using this method appears to follow the intensity pattern of the loops inbetween the two columns, seen in Figure 13, which alternates between red and blueshifts when viewed over time (see Figure 14). This evolves slowly over time, taking about an hour for the blueshifted part to move from one end of the loop to the other. If we instead look at an analysis of the moments of the distributions we find similar intensity and velocity patterns, with values of the same order as those found by Gaussian fitting.

The bottom left-hand panel of Figure 12 shows the velocity along one slit using a single Gaussian fit for both the $\mathrm{Mg} \mathrm{II} \mathrm{k}$ and $\mathrm{h}$ lines. The middle of T2 is around pixel 200, with the centre of $\mathrm{T} 1$ being around pixel 600 . The bottom of the slit corresponds to pixel 0 . There is a velocity gradient around T2, but this is not enough evidence in support of rotation. Around T1 we see large spikes of velocity, which is where the Gaussian fitting fails for the most reversed profiles.

Looking at the spectral images and individual profiles, we notice some undulation in both the blue and red wings along one slit (Figure 11 and profiles from the top of the spectra). These undulations are due to variations in the relative blue and red wing intensities, leading to Doppler shifts on the order of $10 \mathrm{~km} \mathrm{~s}^{-1}$. Some profiles are also shifted by up to $20 \mathrm{~km} \mathrm{~s}^{-1}$ (Figure 11. T1 - 15), however these are transient and do not remain for longer than one or two rasters.

\section{DISCUSSION}

Solar tornadoes and tornado-like prominences have been the topic of a number of publications recently, especially since the launch of the $S D O$ spacecraft with its high-resolution imager AIA. Spectrometers such as those on the Hinode and
IRIS spacecraft have furthered our understanding of these features, but they have also raised many more questions themselves.

We have here shown results from a coordinated observation of a solar tornado from 15 July 2014 . We see two tornado-like features on the limb, and we have presented analysis of this event using data from a number of instruments - SDO/AIA, Hinode/SOT, IRIS, and THEMIS.

Time-distance analysis of our event using SDO/AIA shows periods of around $1-1.5$ hours (Section 2.2.2 and Figure 5). If the structure is rotating, this would give velocities of around $10 \mathrm{~km} \mathrm{~s}^{-1}$, a similar order as that found by $\mathrm{Su}$ et al. (2012). However, Panasenco et al. (2014) argue that such apparent rotation could be in fact oscillation projected onto the plane of the sky, an explaination that we cannot rule out with this data set.

We have presented magnetic field inversions from THEMIS using the $\mathrm{He} \mathrm{I} \mathrm{D}_{3}$ lines. From these inversions we can gain information about the magnetic field strength and orientation inside the prominence structure. We find the strongest magnetic fields (up to $50 \mathrm{G}$ ) in the prominence legs. We also find that the magnetic field is mostly horizontal (parallel to the limb) in these tornado-like columns.

These observations do not fit with the vertical magnetic field structure that has been suggested by previous authors, pointing more towards the horizontal field structure which was explored by Dudík et al. (2012). Luna et al. (2015) have recently suggested a model of a twisted, or 'tornado-like', magnetic field structure with a small slope to the spiral. Field lines close to the axis are mostly vertical, while the field lines close to the edge of the structure are helicoidal. From the inversion of the Stokes profiles we find a nearly constant LOS azimuth of $60^{\circ}$ in T2, while it is varying in T1 (Fig. 6). In both cases, the inversion is affected by the 180 degree ambiguity. Hence we can confirm that we observe a horizontal magnetic field, but we do not have supporting evidence concerning the presence of the mixed azimuth distribution that is suggested by Luna et al. (2015).

Analysis has been done on data from the 15 July 2014 using the IRIS spacecraft. We mostly present analysis from the $\mathrm{Mg}$ II $\mathrm{k}$ and $\mathrm{h}$ lines, although we also make use of the Si IV and $\mathrm{C}$ II lines. We find that the bright prominence legs that are visible in $\mathrm{H}-\alpha$ (from ground-based observatories), IRIS Si IV and $\mathrm{Ca}$ II (from Hinode/SOT) are not readily visible in the $\mathrm{Mg}$ II lines.

We have presented line parameters and intensity ratios from analysis of moments of the Mg II profiles in Table 1 . FWHM values are consistent with predictions from radiative transfer models such as those of Paletou et al. (1993) and Heinzel et al. (2014), as well as previous observations by Vial (1982). Our findings show broader profiles than were found by Schmieder et al. (2014b) using IRIS, however we note that in that paper the $\mathrm{Mg}$ II profiles were not reversed and they did not observe the same dark features in AIA images.

Also shown in Table 1 are intensity ratios of peak intensity to intensity at central reversal $\left(\mathrm{I}_{\text {peak }} / \mathrm{I}_{0}\right)$ for the $\mathrm{Mg}$ II k line, and $k$ to $h$ intensity ratio $\left(I_{k} / I_{h}\right)$. The value given by $I_{\text {peak }} / I_{0}$ tells us about the level of reversal in the profile. Here we find that the profiles in $\mathrm{T} 1$ are generally more reversed than in $\mathrm{T} 2$. The $\mathrm{k}$ to $\mathrm{h}$ intensity ratio is as expected - models such as Paletou et al. (1993) quote a $\mathrm{k} / \mathrm{h}$ ratio of around 1.35, and from Heinzel et al. (2014) it is expected to be around 1.37 1.44. Our values also match well with previous observations, such as those in Vial (1982) and Schmieder et al. (2014b). 

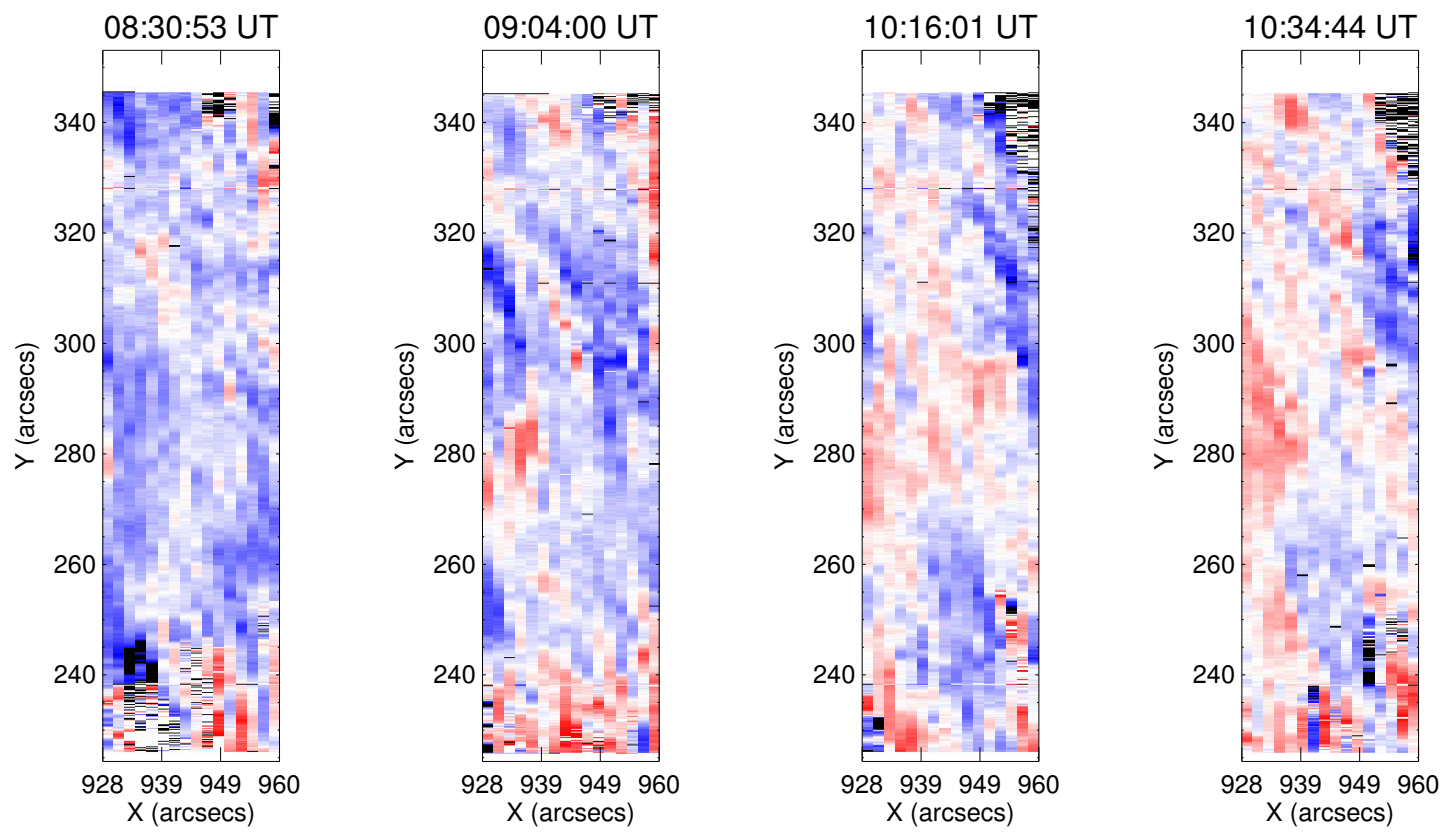

Figure 13. IRIS line-of-sight velocity maps at four different times during the study, made using the Gaussian assumption for the Mg II $\mathrm{k}$ line. Limits for the velocity in these plots are $\pm 10 \mathrm{~km} \mathrm{~s}^{-1}$.
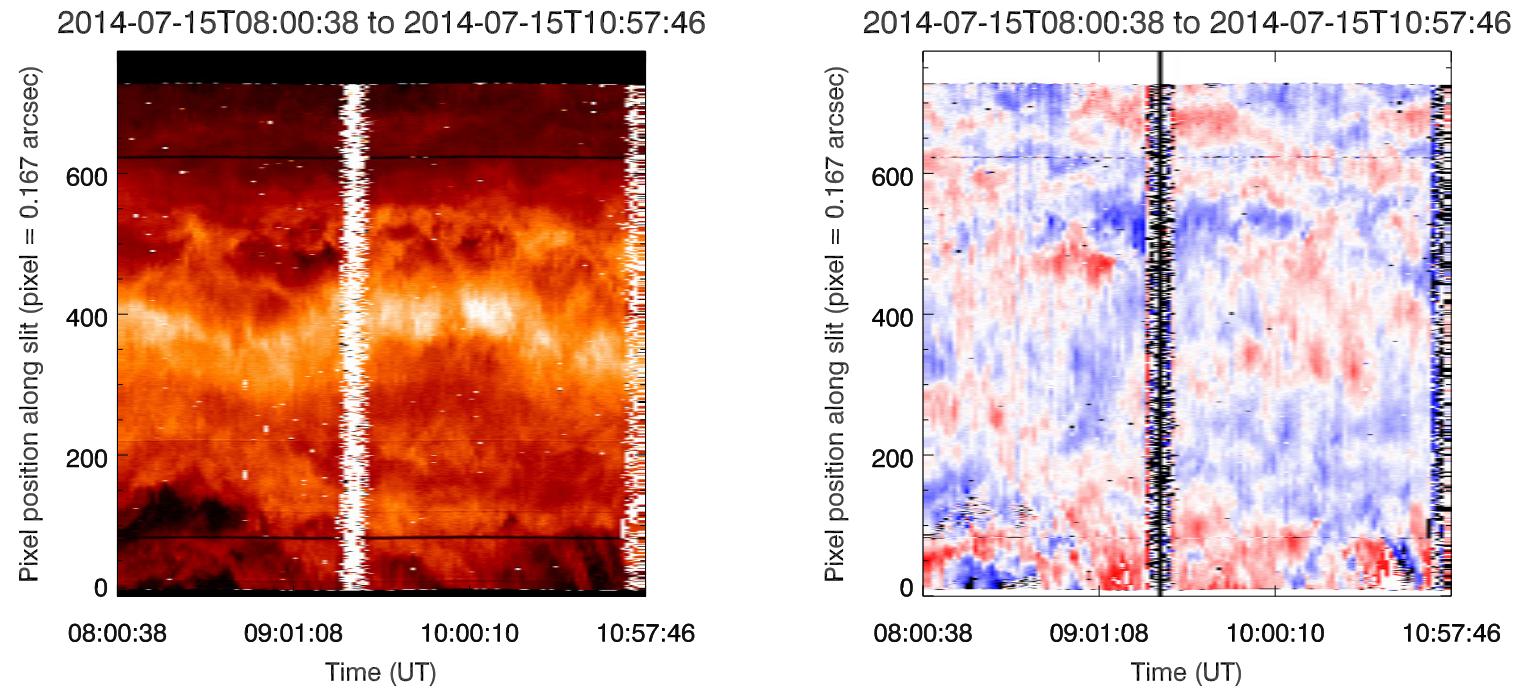

Figure 14. Time evolution of the peak intensity (left) and velocity (right) along the central slit of the IRIS raster. The tornadoes T1 and T2 are located between pixels 535 and 650, and 190 and 260 respectively. The vertical noise dominated regions are caused by the spacecraft passing over the South Atlantic Anomaly. 
The level of reversal of the profile allows us to compare with models in order to constrain the gas pressure and optical thickness of the prominence. Comparing our results to a 1D isothermal-isobaric radiative transfer model (Heinzel et al. 2014 table 2), for a prominence slab of geometrical thickness of $1000 \mathrm{~km}$ and temperature $6000 \mathrm{~K}$, we get a gas pressure, $\rho$, of $0.1-0.5$ dyne $\mathrm{cm}^{-2}$ and an optical thickness, $\tau_{\mathrm{Mg}}$, of 140 -820 . However, for T2 (corresponding to the lower limit of $\rho$ and $\tau_{\mathrm{Mg}}$ ) we note that the absorption in AIA $171 \AA$ is strong, as is the emission in $\mathrm{Ca}$ II and $\mathrm{He} \mathrm{I} \mathrm{D}_{3}$. We therefore must consider $2 \mathrm{D}$ models which contain a prominence-to-corona transition region.

We have analysed line-of-sight velocities in the $\mathrm{Mg}$ II line (formed at chromospheric temperatures) from IRIS, to study Doppler velocities at lower plasma temperatures. Previously, Su et al. (2014) and Levens et al. (2015) presented results showing a split Doppler pattern in a tornado-like prominence at coronal temperatures ( $>1 \mathrm{MK})$. Orozco Suárez et al. (2012) and Wedemeyer et al. (2013) showed a similar Doppler pattern in the He I $10830 \AA$ A and $\mathrm{H}-\alpha$ lines respectively, but little work has been done on lines formed at temperatures of $\log \mathrm{T}=4.5$. These results are consistent with what has been obtained in other studies comparing optically thick lines with various radiative transfer models (Labrosse et al. 2010).

In the Doppler maps of Mg II k we find no such split pattern. We see redshifts and blueshifts on the order of \pm 10 $\mathrm{km} \mathrm{s}^{-1}$, but these seem to be associated with the loop-like structure that is seen in intensity maps of this ion (Figure 13). There is also no consistant split pattern when we look at the evolution over time (Figure 14). These results do not support any of the rotating 'tornado' models, however we must acknowledge that the $\mathrm{Mg}$ II $\mathrm{k}$ and $\mathrm{h}$ lines are optically thick (as discussed in Section 3.2.1). This means that the radiation we receive is only from the frontmost layers of the prominence, as opposed to integrated all the way along the line of sight through the tornado structure as in the optically thin case.

\section{CONCLUSIONS}

We have presented results from a coordinated prominence observation on 15 July 2014 using the SDO/AIA, IRIS and Hinode/SOT instruments as well as the THEMIS spectropolarimeter. The prominence studied here was chosen due to its tornado-like nature, and good coordination between the instruments covering two tornado-like prominence legs.

The prominence legs appear as dark, silhouetted features in AIA coronal filters $(171 \AA, 193 \AA$ etc. $)$ due to the absorption of background emission by material contained in the prominence. These columns appear bright in IRIS Si IV, Hinode/SOT Ca II and THEMIS He $\mathrm{I} \mathrm{D}_{3}$ lines, but we see no such brightenings in IRIS Mg II line profiles. We do, however, find central reversal at these positions in $\mathrm{Mg}$ II which is not present elsewhere in the raster.

Comparing $\mathrm{Mg}$ II profiles observed by IRIS to those expected from radiative transfer models, both 1D (Heinzel et al. 2014) and 2D (Paletou et al. 1993), we find a good agreement with our data. These models allow us to constrain physical parameters of the plasma that we observe. We recover gas pressures of around $0.1-0.5$ dyne $\mathrm{cm}^{-2}$ and high optical thicknesses of between 140 and 820 for the $\mathrm{Mg}$ II k and h lines. Qualitatively, the best agreement is obtained with 2D models which contain a prominence-to-corona transition region.

We performed line-of-sight velocity analysis of the Mg II $\mathrm{k}$ line and found no evidence for rotation in this line. Time- distance analysis of AIA images reveal that there are oscillations in the two tornadoes over a period of around $1-1.5$ hours. It is not possible to conclude what mechanism causes these oscillations from these observations.

THEMIS provides magnetic field information in these structures, both orientation and field strength. We recover field strengths that are strongest in the two tornado columns, on the order of $20-50 \mathrm{G}$. The inclination of the field tells us that the field is horizontal everywhere, parallel to the limb. The line-of-sight azimuth reveals values of between $70^{\circ}$ and $100^{\circ}$.

Coordinated observation between THEMIS and other instruments provide us with a unique opportunity to study the magnetic field and plasma properties in prominence legs. We plan to extend our investigation of these dynamic structures in a future paper.

The authors thank S. Gunar, B. Gelly and the team of THEMIS for assisting with the observations. The authors also thank the anonymous referee for their comments which helped improve the clarity of this paper. P.J.L. acknowledges support from an STFC Research Studentship ST/K502005/1. N.L. acknowledges support from STFC grant ST/L000741/1. Hinode is a Japanese mission developed and launched by ISAS/JAXA, with NAOJ as domestic partner and NASA and STFC (UK) as international partners. It is operated by these agencies in co-operation with ESA and NSC (Norway). IRIS is a NASA small explorer mission developed and operated by LMSAL with mission operations executed at NASA Ames Research center and major contributions to downlink communications funded by the Norwegian Space Center (NSC, Norway) through an ESA PRODEX contract. The AIA data are provided courtesy of NASA/SDO and the AIA science team.

\section{REFERENCES}

Aulanier, G. \& Démoulin, P. 1998, A\&A, 329, 1125

Casini, R., Bevilacqua, R., \& López Ariste, A. 2005, ApJ, 622, 1265

Casini, R., López Ariste, A., Tomczyk, S., \& Lites, B. W. 2003, ApJL, 598, L67

Culhane, J. L., Harra, L. K., James, A. M., et al. 2007, Sol. Phys., 243, 19

De Pontieu, B., Title, A. M., Lemen, J., et al. 2014, ArXiv e-prints

Dere, K., Landi, E., Mason, H., Monsignori Fossi, B., \& Young, P. 1997, A+A Suppl. Ser., 125, 149

Domingo, V., Fleck, B., \& Poland, A. I. 1995, Sol. Phys., 162, 1

Dudík, J., Aulanier, G., Schmieder, B., Bommier, V., \& Roudier, T. 2008, Sol. Phys., 248, 29

Dudík, J., Aulanier, G., Schmieder, B., Zapiór, M., \& Heinzel, P. 2012, ApJ, 761,9

Heinzel, P., Schmieder, B., Mein, N., \& Gunár, S. 2015, ApJL, 800, L13

Heinzel, P., Vial, J.-C., \& Anzer, U. 2014, A\&A, 564, A132

Kosugi, T., Matsuzaki, K., Sakao, T., et al. 2007, Sol. Phys., 243, 3

Labrosse, N. \& Gouttebroze, P. 2001, A\&A, 380, 323

Labrosse, N. \& Gouttebroze, P. 2004, ApJ, 617, 614

Labrosse, N., Heinzel, P., Vial, J.-C., et al. 2010, Space Sci. Rev., 151, 243

Labrosse, N., Schmieder, B., Heinzel, P., \& Watanabe, T. 2011, A+A, 531, A69

Landi, E., Del Zanna, G., Young, P., Dere, K., \& Mason, H. 2012, A+A Suppl. Ser., 744, 99

Landi Degl'Innocenti, E. \& Landolfi, M., eds. 2004, Astrophysics and Space Science Library, Vol. 307, Polarization in Spectral Lines

Lemen, J. R., Title, A. M., Akin, D. J., et al. 2012, Sol. Phys., 275, 17

Levens, P. J., Labrosse, N., Fletcher, L., \& Schmieder, B. 2015, A\&A, 582, A27

Li, X., Morgan, H., Leonard, D., \& Jeska, L. 2012, ApJ Letters, 752, L22

López Ariste, A. 2014, in Proceedings of the International Astronomical Union, Vol. 10, Polarimetry: From the Sun to Stars and Stellar Environments, 207-215

López Ariste, A., Asensio Ramos, A., Manso Sainz, R., Derouich, M., \& Gelly, B. 2009, A\&A, 501, 729 
López Ariste, A., Aulanier, G., Schmieder, B., \& Sainz Dalda, A. 2006, A\&A, 456, 725

López Ariste, A. \& Casini, R. 2002, ApJ, 575, 529

López Ariste, A., Rayrole, J., \& Semel, M. 2000, A\&AS, 142, 137

Luna, M., Moreno-Insertis, F., \& Priest, E. 2015, ApJL, 808, L23

Mackay, D. H., Karpen, J. T., Ballester, J. L., Schmieder, B., \& Aulanier, G. 2010, Space Sci. Rev., 151, 333

Orozco Suárez, D., Asensio Ramos, A., \& Trujillo Bueno, J. 2012, ApJL, 761, L25

Paletou, F., Vial, J.-C., \& Auer, L. H. 1993, A\&A, 274, 571

Panasenco, O., Martin, S. F., \& Velli, M. 2014, Sol. Phys., 289, 603

Panesar, N. K., Innes, D. E., Tiwari, S. K., \& Low, B. C. 2013, A+A, 549, A105

Pettit, E. 1932, ApJ, 76, 9

Pettit, E. 1943, ApJ, 98, 6

Pike, C. D. \& Mason, H. E. 1998, Sol. Phys., 182, 333
Scharmer, G. B., Bjelksjo, K., Korhonen, T. K., Lindberg, B., \& Petterson, B. 2003, in Society of Photo-Optical Instrumentation Engineers (SPIE) Conference Series, Vol. 4853, Innovative Telescopes and Instrumentation for Solar Astrophysics, ed. S. L. Keil \& S. V. Avakyan, 341-350

Schmieder, B., Kucera, T. A., Knizhnik, K., et al. 2013, ApJ, 777, 108

Schmieder, B., López Ariste, A., Levens, P., Labrosse, N., \& Dalmasse, K. 2014a, in IAU Symposium, Vol. 305, IAU Symposium, 275-281

Schmieder, B., Tian, H., Kucera, T., et al. 2014b, A\&A, 569, A85

Su, Y., Gömöry, P., Veronig, A., et al. 2014, ApJ Letters, 785, L2

Su, Y., Wang, T., Veronig, A., Temmer, M., \& Gan, W. 2012, ApJ Letters, 756, L41

Suematsu, Y., Tsuneta, S., Ichimoto, K., et al. 2008, Sol. Phys., 249, 197

Tsuneta, S., Ichimoto, K., Katsukawa, Y., et al. 2008, Sol. Phys., 249, 167

Vial, J. C. 1982, ApJ, 253, 330

Wedemeyer, S., Scullion, E., Rouppe van der Voort, L., Bosnjak, A., \& Antolin, P. 2013, ApJ, 774, 123

Wedemeyer, S., Scullion, E., Steiner, O., de la Cruz Rodriguez, J., \&

Rouppe van der Voort, L. H. M. 2013, Journal of Physics Conference Series, 440, 012005

Wedemeyer-Böhm, S., Scullion, E., Steiner, O., et al. 2012, Nature, 486, 505 